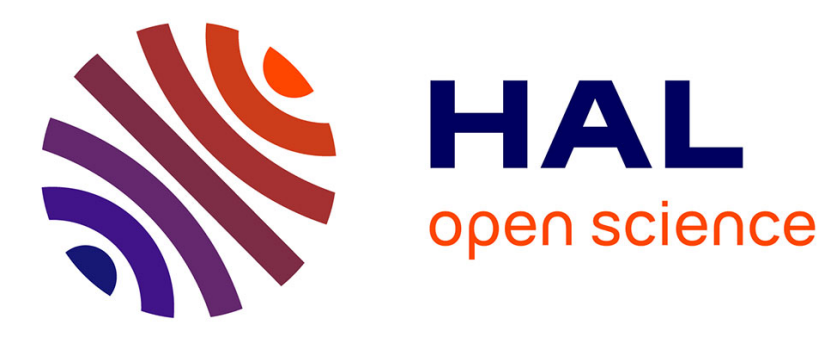

\title{
Time window selection for improving error-related potential detection
}

\author{
Sandra Rousseau, Christian Jutten, Marco Congedo
}

\section{To cite this version:}

Sandra Rousseau, Christian Jutten, Marco Congedo. Time window selection for improving errorrelated potential detection. NCTA 2012 - 5th International Conference on Neural Computation Theory and Applications, Oct 2012, Barcelone, Spain. pp.1. hal-00741234

\section{HAL Id: hal-00741234 \\ https://hal.science/hal-00741234}

Submitted on 12 Oct 2012

HAL is a multi-disciplinary open access archive for the deposit and dissemination of scientific research documents, whether they are published or not. The documents may come from teaching and research institutions in France or abroad, or from public or private research centers.
L'archive ouverte pluridisciplinaire $\mathbf{H A L}$, est destinée au dépôt et à la diffusion de documents scientifiques de niveau recherche, publiés ou non, émanant des établissements d'enseignement et de recherche français ou étrangers, des laboratoires publics ou privés. 


\title{
Time window selection for improving error-related potential detection
}

\author{
Rousseau Sandra ${ }^{1}$, Jutten Christian ${ }^{1}$ and Congedo Marco ${ }^{1}$ \\ ${ }^{1}$ Gipsa-lab-DIS, 11 rue des mathématiques Domaine Universitaire, 38400 Saint-Martin d'Héres, France \\ sandra.rousseau@gipsa-lab.grenoble-inp.fr
}

Keywords: Error-related potential, BCI, Spatial filtering, Classification

\begin{abstract}
In this paper we present an experiment enabling the occurence of the error-related potential in high cognitive load conditions and observe its inter-subject latency variability. We study the single-trial classification of the error-related potential using spatial filtering. Then we present a new adaptive algorithm for spatial filtering and time window selection that allows to adapt to error-related potential latency variability and provides better classification results.
\end{abstract}

\section{Introduction}

The error-related potential (ErrP) is an eventrelated potential (ERP) which is generated when a subject commits or observes the commitment of an error. It was first reported in 1991 by Falkenstein et al. (Falkenstein et al., 1991) and has been since the subject of growing interest. This potential is time-locked to the observation of the error and is mostly characterized by a negative deflection (Ne) (Gentsch et al., 2009), followed by a large positivity (Pe) (Steinhauser and Kiesel, 2011). There exists different kind of ErrPs depending on the agent committing the error. In BCIs we generally observe what is called the interaction ErrP which occurs when a subject observe an external device committing an error and the feedback ErrP which is observed when a subject commits an error but becomes aware of it only after external feedback. Lately several authors have become interested in its integration in BCI systems as a control loop, for example as an error correction system. The integration of the ErrP in BCIs involves two main operations: its single trial detection and the use of this information to on-line modify the system. Since its signal to noise ratio (SNR) is very low, as any ERP, the ErrP can easily be seen by summing up several trials but is much less detectable on a single-trial basis. However, the single trial detection of the ErrP is mandatory for its integration in BCIs. In addition to its low SNR, ErrP characteristics can also be highly variable from one subject to another and from one experiment to another. Thus to ensure satisfying detection rates, it is necessary to design filtering methods that will increase the ErrP SNR. Moreover, designing subject's specific fil- ters could improve detection accuracy. In this paper, we first present an experiment we designed to obtain ErrP data in high cognitive load conditions, enabling us to approximate BCI use conditions. We study the occurrence of the ErrP and observe its inter-subject latency variability. Then we classify this potential on a single-trial basis using spatial filtering methods. Based on our observations of the inter-subject latency variability we develop a new adaptive spatial filtering method which allows the selection of subjects specific time windows in order to improve single trial detection results. Our method aims at selecting subjects specific time windows based on each subject signal characteristics and optimized for the spatial filter we use. We compare the classification results we obtain with other less adaptive methods. Finally we comment on the relevance of the selected time window and its link with our signals characteristics.

\section{Data}

\subsection{Experiment}

The experiment we designed involves a memory game where performance feedback is given after the subject answers questions. No specific reward is given and the experiment is designed so as to induce a high cognitive load. The experiment involves two sessions that last approximately half an hour. Each session consists of six blocks of six trials, for a total of 72 trials. Stimuli are presented on a computer screen and consist of digits displayed in square boxes arranged in circles. 


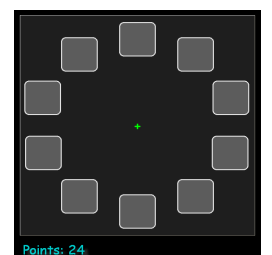

(a)

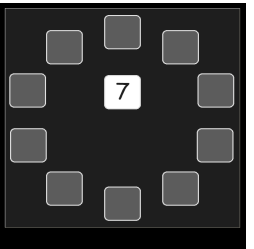

(c)

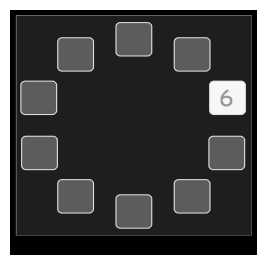

(b)

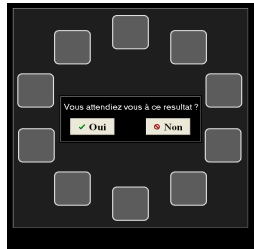

(d)
Figure 1: Screen shots from the experiment representing different steps.a)Fixation cross. b)Memorization sequence. c)Target digit appearing. d) Feedback report question: 'Did you expect this result?'.

Each trial starts with the display of the score for $3000 \mathrm{~ms}$ followed by a fixation cross (see Figure 1.a) also displayed for $3000 \mathrm{~ms}$. Then the memorization sequence starts, each memorization consists in a random sequence of two to nine digits appearing sequentially in random positions (see Figure 1.b). Subjects are instructed to retain positions of all digits. At the end of the sequence the target digit is displayed (see Figure 1.c) and subjects have to click on the box where it has appeared. Once the subject has answered, the interface waits for $1500 \mathrm{~ms}$ in order to avoid any contamination of ErrP by beta rebound motor phenomena linked to mouse clicking (Pfurtscheller, 1981). Then, if the answer is correct, the chosen box background color turns into green ("correct" feedback), otherwise it turns into red ("error" feedback). Subjects are then asked to report if the feedback (error/correct) matches their expectation by a mouse click ("yes"/ "no") (see Figure 1.d). A random break of $1000 \mathrm{~ms}$ to $1500 \mathrm{~ms}$ precedes the beginning of the new trial (see Figure 2).

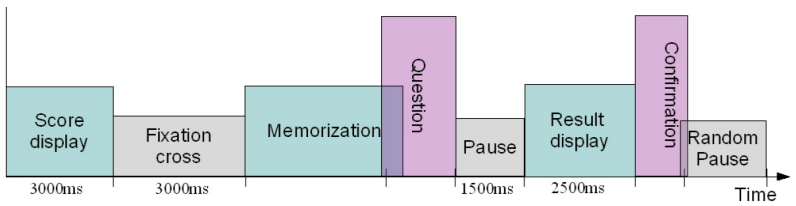

Figure 2: Time course of one trial

The number of digits in the sequence is fixed within blocks (between two and nine) and updated, according to subjects performance, at the beginning of each block. The first block starts with four digits. The number of digits is adapted with an algorithm tuned to allow about $20 \%$ of errors for all subjects, regardless the working memory ability and adapting to fatigue. Between the two sessions the screen is shut down to allow a rest break of 2 - 3 minutes.

\subsection{Participants}

25 healthy volunteer subjects participated, 14 males and 11 females. Subjects were informed of the procedure before the experiment and filled an information form. All subjects were BCI-naifs at the time of the experiment and none of them reported neurological or psychiatric disorders in the past. Due to the presence of artifacts, four subjects were excluded from analysis. The age of participants ranged from 20 to 30 with a mean of $24( \pm 2.5)$. The mean error rate was equal to $18( \pm 4.6) \%$ of the trials.

\subsection{Acquisition and preprocessing}

EEG recordings were made from 31 sensors using the extended 10/20 system. Both earlobes, digitally linked, were used as electrical reference. The ground sensor was positioned on the forehead. The impedance of each sensor was kept below $5 k \Omega$. The EEG was band-pass filtered in the range 0.1-70 Hz and digitized at $500 \mathrm{~Hz}$ using the Mitsar 202 DC EEG acquisition system. Data were bandpass-filtered between 1-40 Hz using an order 4 Butterworth filter with linear phase response. Eye blinks were extracted using ICA (independent component analysis), (Comon and Jutten, 2010). SOBI algorithm was used to perform ICA. One EOG source was suppressed for each subject. It was manually selected using both the temporal shape of the source and its topography.

\subsection{Observation of the ErrP and its latency variability}

In Figure 3 we plot the event-related potential averaged over subjects for correct trials and for error trials for one second post-stimulus (observation of the error). We can see that for error trials the ERP is characterized by a sharp negativity $(\mathrm{Ne})$ followed by a small positivity $(\mathrm{Pe})$ which is consistent with previous reports (Yeung et al., 2005), (Hajcak et al., 2007). In addition to these peaks one can also see a first positive peak which has also been reported by (Ferrez and Millán, 2005) in the case of interaction error-related potential. For correct trials, a negativity is also observed but with a much lower intensity. Let us define $\mathrm{Ne}$ latency as the time where the mean potential 


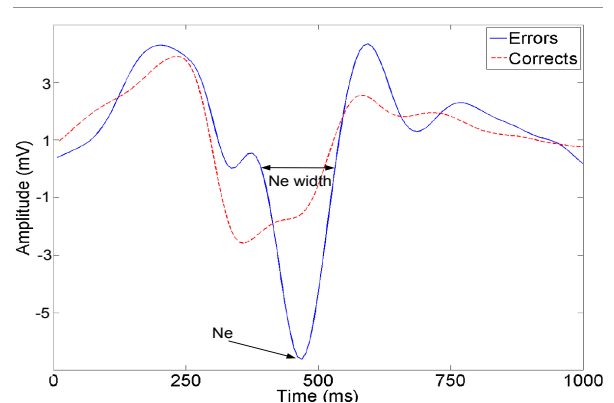

Figure 3: Mean potential averaged over 21 subjects at electrode FCz. Blue thick line corresponds to error trials and red dashed line corresponds to correct trials

reaches a local minimum in the time window [350 ms $650 \mathrm{~ms}$ ] and the peak width as the time separating the last time point where the EEG signal crosses zero before $\mathrm{Ne}$ and the first time point it crosses zero after $\mathrm{Ne}$ as shown in Figure 3. Then the mean Ne latency (over the 21 subjects) and its standard deviation are $490 \mathrm{~ms}$ $\pm 50 \mathrm{~ms}$ while the mean peak width is $110 \mathrm{~ms}$. This shows the high variability of $\mathrm{Ne}$ latency since its variation corresponds to half the width of the peak. In Figure 4 we plot the mean event-related potentials for error trials for three different subjects. We can see that the shape and the latency of this potential can be highly variable.

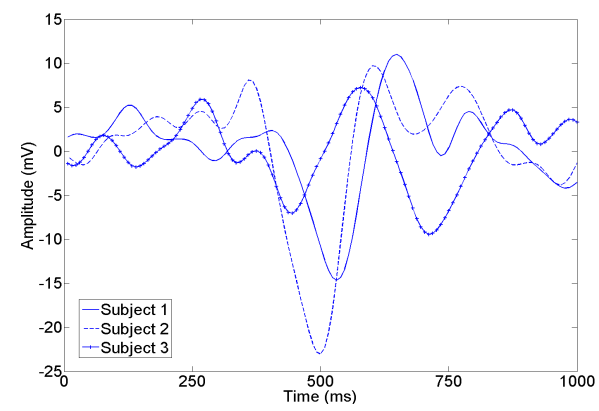

Figure 4: Temporal course of the mean error potential for different subject.Each line corresponds to one subject. Data are averaged over error trials at electrode FCz.

\section{Single trial classification}

ErrP could be used in BCI systems to detect and correct error trials. However, to do so, one has to be able to detect it on a single trial basis. Some authors already studied this issue (Dal Seno et al., 2010),(Ferrez and Millán, 2007). Most of them only used raw data with no spatial filtering. Here we propose to apply spatial filtering methods to our data in order to try to improve our classification results.

\subsection{Filtering method}

\subsection{1 xDAWN algorithm}

Spatial filtering was done using xDAWN algorithm (Rivet et al., 2008). We consider that the signal is the sum of one target evoked potential plus other possible superimposed evoked potentials (but non-target) and noise:

$$
X=\sum_{i} D_{i} A_{i}+N
$$

where $X$ represents the signal, $D_{i}$ is a Toeplitz matrix whose first column entries are set to zero except for those that correspond to the stimuli of type i, $A_{i}$ represents the responses synchronized with the stimuli of type i and $N$ represents the noise. Evoked responses $A_{i}$ are estimated as:

$$
\left(\begin{array}{c}
A_{1} \\
\ldots \\
A_{n}
\end{array}\right)=\left(D^{T} D\right)^{-1} D^{T} X
$$

with $D=\left[D_{1}, D_{2} \ldots D_{n}\right]$.

The aim of $x D A W N$ is to find the filter $U$ which maximizes the signal to signal plus noise ratio (SSNR):

$$
U=\operatorname{argmax} \frac{\operatorname{Tr}\left(U^{T} \sum_{i} U\right)}{\operatorname{Tr}\left(U^{T} \sum_{X} U\right)}
$$

with $\sum_{i}=\left(D_{i} A_{i}\right)^{T}\left(D_{i} A_{i}\right)$ and $\sum_{X}=X^{T} X$. This is a Rayleigh quotient ((Fukunaga, 1990), (Gray, 2006)) which can be solved using the generalized eigenvalue decomposition (GEVD) of matrices $\Sigma_{1}$ and $\Sigma_{X}$.

\subsubsection{Application to our problem}

In this problem we consider two potentials: one corresponding to the occurrence of an error (the error potential) and one corresponding to the occurrence of answer display (may it be correct or error). Thus our signal can be written as :

$$
X=D_{1} A_{1}+D_{2} A_{2}+N
$$

where $D_{1} A_{1}$ corresponds to error feedbacks and $D_{2} A_{2}$ corresponds to feedbacks (errors and corrects). The evoked potentials are usually defined as a one second post-stimulus window of the EEG signal. However, the longer will be this window and the more our potential will contain noise. Thus, it could be interesting to reduce the size of this window. One way of doing it is to determine the position of our peak of interest (defined here as $\mathrm{Ne}$ ) and to select a smaller time window centered on this peak. However as we have seen before Ne latency can be highly variable from one subject to another, making it impossible to select a prior time window that could be used for all subjects. 


\subsection{Adaptive time window selection}

We propose a modification of XDAWN that allows a subject's specific selection of the time window within the spatial filtering algorithm. Here we span the whole ERP and find the time window where the target potential (error trials) contains the most information. The time-window will then be used to estimate the spatial filters and to classify data. Let be $L$ the length of the window of interest (here set to $250 \mathrm{~ms}$ ), $N$ the maximal length of the potential under study (here $1000 \mathrm{~ms}$ ) and $x$ the EEG signal of total length $N_{T}$ with $N_{c}$ electrodes. Then the proposed algorithm is as follows:

We first calculate the matrices $A_{1} \in R^{N \times N_{c}}, D_{1} \in$ $\mathcal{R}^{N_{T} \times N}$ and $\Sigma_{X} \in \mathcal{R}^{N_{c} \times N_{c}}$ then $\forall t \in[0, . . N-L]$ :

- We create matrices $D_{1 \text { new }}$ et $A_{1 \text { new }}$ defined so that they contain only the signal corresponding to the window of length $\mathrm{L}$ starting at time point $t$ after the feedback:

$D_{\text {lnew }}=D_{1}(:, t: t+L)$ and $A_{1 \text { new }}=A_{1}(t: t+L,:)$;

- We create matrix $\Sigma_{1 \text { new }}$ defined as :

$$
\Sigma_{\text {new }}=\left(D_{1 \text { new }} \cdot A_{1 \text { new }}\right)^{\prime}\left(D_{1 \text { new }} \cdot A_{1 \text { new }}\right) \text {; }
$$

- We compute the generalized eigenvalue decomposition (GEVD) of $\Sigma_{1 \text { new }}$ and $\Sigma_{X}$

$$
\left(U_{t}, \lambda\right)=\operatorname{GEV} D\left(\Sigma_{\text {1new }}, \Sigma_{X}\right) ;
$$

- Finally we define our decision value as:

$$
\beta(t)=\lambda(1)
$$

with $\lambda(1)$ being the first eigenvalue of the GEVD.

The optimal position of our window $\left(t_{o p t}\right)$ is then defined as the time sample corresponding to the maximum of $\beta$. Thus, for spatial filtering we will use filters computed on this time window $\left(U_{t_{o p t}}\right)$.

\subsection{Preprocessing and classification}

Data were band-pass filtered between $1-10 \mathrm{~Hz}$ and then spatially filtered using the previously described methods. Data were then classified using Bayesian LDA classifier (MacKay, 1992). xDAWN algorithm returns filters classified in descending order of performance thus we used the first two components as features for our classifier. Data were subsampled at $32 \mathrm{~Hz}$. Data were also classified before spatial filtering using EEG signal from electrodes $\mathrm{FCz}$ and $\mathrm{Cz}$ as features, as it has been done in most previous studies (Ferrez and Millán, 2007). Classification was performed on 21 subjects. For each subjects a leave-one out validation method was used: spatial filters and classifier were learned on the whole data except one and then tested on the remaining data.

\section{Results}

\subsection{Evaluation of the algorithm}

In figure 5 we plot the evolution of our decision value $(\beta)$ along our window position and the evolution of our mean classification accuracy. We can see that $\beta$ is highly influenced by the window position. Moreover we can also see that $\beta$ and our classification accuracy seem to have a similar evolution. Thus it seems that the choice of our decision value is relevant for the optimization of the single trial detection of the ErrP.
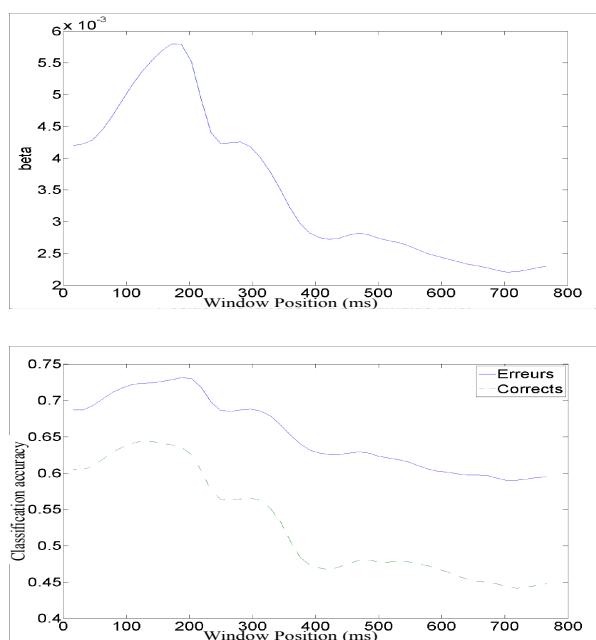

Figure 5: Effect of window position.

(a)Evolution of $\beta$ as a function of window position. (b)Evolution of classification accuracy as a function of window position. Thick line corresponds to errors, dashed line corresponds to corrects.

\subsection{Selection of the time window}

In figure 6 we plot the mean error potential for three different subjects and the corresponding selected time window. We can see that for these three subjects the shape and latency of the ErrP is very different and that our algorithm adapts to the shape of the potential under study and select the time window where the difference between errors and corrects is huger.

\subsection{Classification Results}

We report the mean classification results (and their standard deviation) on table 1 for our three different methods. It has to be noted that given the very low number of trials available we get quite encouraging results even on raw data.

Secondly we can see that XDAWN allows significantly better classification results for correct trials 


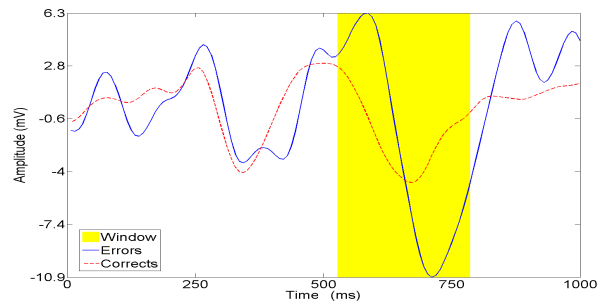

(a) Subject 18

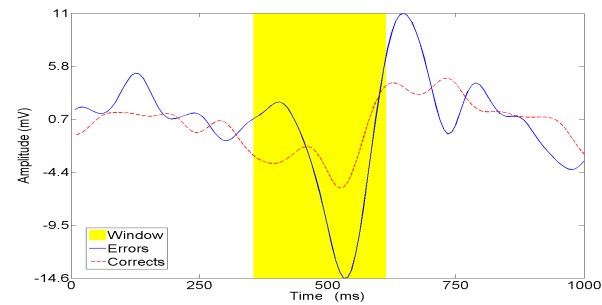

(b) Subject 7

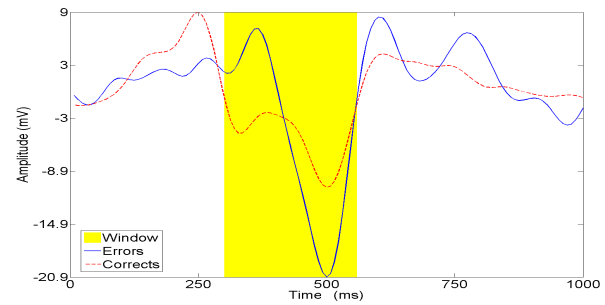

(c) Subject 5

Figure 6: Selected time windows for different subjects. Yellow area corresponds to the selected time window. Thick line is the averaged potential for errors and dashed line is the averaged potential for corrects, at electrode $\mathrm{FCz}$.

\begin{tabular}{|c|c|c|c|c|c|}
\hline Method & $T_{e}$ & std & $T_{c}$ & std & $\frac{T_{e}+T_{c}}{2}$ \\
\hline No filtering & 66 & \pm 14 & 65 & \pm 10 & 65.5 \\
\hline xDAWN & 56 & \pm 16 & 81 & \pm 7 & 68.5 \\
\hline New xDAWN & 67 & \pm 19 & 82 & \pm 6 & 74.5 \\
\hline
\end{tabular}

Table 1: Mean classification results.

First line corresponds to classification on raw data, second line to classification with XDAWN and third line to classification with our adaptive xDAWN. Column $T_{e}$ gives the classification rate for errors and column $T_{c}$ for corrects.

than classification on raw data $(p \leq 0.01)$. However for classification on error trials XDAWN is worse than using raw data $(p=0.01)$. Thus even if XDAWN improves the mean classification rate $\left(\frac{T_{e}+T_{c}}{2}\right)$, it gives very poor results for error classification. However for most types of ErrP integration, correct trials identification is more important than error trials identification. A misidentification of a correct trial (correct trial is identified as an error trial) will lead to the modification of a correct order (and thus leading to an error) which will diminish our system's performance. The misidentification of an error will just lead to no specific action, which will bring no modification.

Finally it turns out that our adaptive algorithm gives similar results as the classical xDAWN for correct trials $(p=0.2)$ (and thus significantly better than raw data, $p \leq 0.01$ ) but significantly better results for error trials $(p \leq 0.01)$ (and similar as raw data, $p=0.3$ ). The main advantage of our method is that it is optimized for the spatial filter we use and each time window is selected specifically for one subject. Our algorithm allows a significant improvement of detection of correct trials along with no deterioration of error trials detection (but no improvement) as compared to classification on raw data. Using this method we get similar results as those found by previous studies ((Chavarriaga and Millán, 2010),(Ferrez and Millán, 2007)) but with only very small training sets while other studies used large training sets. This is of great interest for ErrP integration in BCIs. Indeed one huge problem in actual BCI systems is the training session which can be quite long for some applications. Here we get significant detection results even when using only 15 error trials (30 trials in total, errors and corrects) which would correspond to a very short training session.

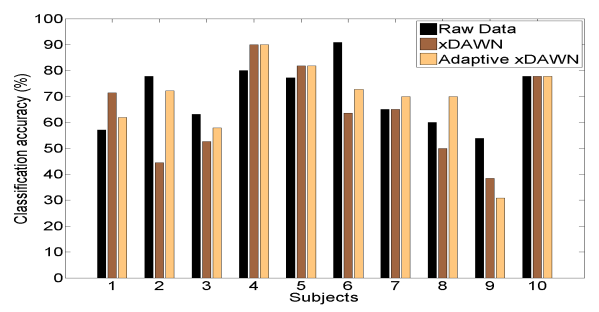

(a) Error trials

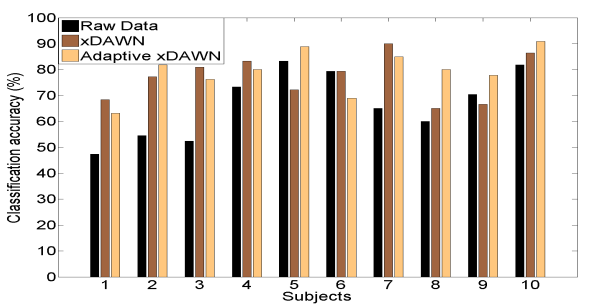

(b) Correct trials

Figure 7: Classification results for the first ten subjects. (a) for error trials. (b) for correct trials. Black bars correspond to raw data, dark brown bars to xDAWN filtering and light brown bars to adapative xDAWN filtering.

In figure 7 we plot the classification results for the ten first subjects for error trials and correct trials using the different preprocessing methods. We can see that for classification on error trials xDAWN only outperforms our adaptive algorithm for two subjects and 
for one of them results are very bad for any method. For correct trials spatial filtering clearly outperforms classification on raw data, however no clear difference can be drawn between our two methods, with xDAWN outperforming the adaptive one for half the subjects.

\section{Conclusion}

In this paper we have studied the error-related potential and its single-trial detection. We have seen that the ErrP did occur even in high cognitive load conditions and that its latency could be highly variable from one subject to another. Based on this observation we have proposed a new algorithm for adaptive spatial filtering and time window selection based on xDAWN algorithm. Classification results have shown that our algorithm performs better than the xDAWN algorithm and than classification on raw data with an improvement up to $17 \%$ for correct trials (which is the most important one as we have said before). Thus, we have developped a new algorithm that allows our classifier to adapt to ErrP latency variability. This is of great interest given its high inter-subject variability. Moreover it is known that ErrP characteristics may vary with experimental and environmental conditions. ErrP latency could then evolve within an experiment, using an adaptive filtering method (that would be updated along the experiment) would allow consistent classification results along time. In conclusion we have shown that using adaptive filters for ErrP single trial detection could significantly improve classification results. Moreover we have developped a new algorithm that could tackle the problem of ErrP latency variability. Further studies should be done in this way, as for example to adapt to latency variability between trials or maybe to find otpimal time windows for the different ErrP peaks (since it is known that the ErrP is characterized by a negative peak and a positive one).

\section{ACKNOWLEDGEMENTS}

We are grateful to Dr. Bertrand Rivet for making us available the code of his xDAWN algorithm and explaining us his method. We are grateful to the project ANR OpenVibe.

\section{REFERENCES}

Chavarriaga, R. and Millán, J. (2010). Learning from EEG error-related potentials in noninvasive brain-computer interfaces. IEEE Trans Neural Syst Rehabil Eng, 18(4):381-388.

Comon, P. and Jutten, C. (2010). Handbook of Blind Source Separation: Independent component analysis and applications. Academic Press.

Dal Seno, B., Matteucci, M., and Mainardi, L. (2010). Online detection of $\mathrm{P} 300$ and error potentials in a BCI speller. Computational intelligence and neuroscience, 2010:1-1.

Falkenstein, M., Hohnsbein, J., Hoormann, J., and Blanke, L. (1991). Effects of crossmodal divided attention on late ERP components.II. Error processing in choice reaction tasks. Electroencephalogr. Clin. Neurophysiol., 78:447-455.

Ferrez, P. and Millán, J. (2005). You are wrong!—automatic detection of interaction errors from brain waves. In Proceedings of the 19th International Joint Conference on Artificial Intelligence, 2005.

Ferrez, P. and Millán, J. (2007). EEG-based brain-computer interaction: Improved accuracy by automatic singletrial error detection. In Proc. NIPS 20.

Fukunaga, K. (1990). Introduction to statistical pattern recognition (2nd ed.). Academic Press Professional, Inc., San Diego, CA, USA.

Gentsch, A., Ullsperger, P., and Ullsperger, M. (2009). Dissociable medial frontal negativities from a common monitoring system for self-and externally caused failure of goal achievement. Neuroimage, 47(4):20232030.

Gray, R. M. (2006). Toeplitz and circulant matrices: A review. Foundations and Trends in Communications and Information Theory, 2(3):155-239.

Hajcak, G., Moser, J., Holroyd, C., and Simons, R. (2007). It's worse than you thought: The feedback negativity and violations of reward prediction in gambling tasks. Psychophysiology, 44(6):905-912.

MacKay, D. (1992). Bayesian interpolation. Neural computation, 4(3):415-447.

Pfurtscheller, G. (1981). Central beta rhythm during sensorimotor activities in man. Electroencephalography and clinical Neurophysiology, 51(3):253-264.

Rivet, B., Souloumiac, A., Gibert, G., and Attina, V. (2008). P300 speller brain-computer interface: Enhancement of P300 evoked potential by spatial filters. In Proceedings of the 16th European Signal Processing Conference (EUSIPCO-2008), EURASIP, Lausanne, Switzerland.

Steinhauser, M. and Kiesel, A. (2011). Performance monitoring and the causal attribution of errors. Cognitive, Affective, \& Behavioral Neuroscience, pages 1-12.

Yeung, N., Holroyd, C., and Cohen, J. (2005). ERP correlates of feedback and reward processing in the presence and absence of response choice. Cerebral Cortex, 15(5):535. 